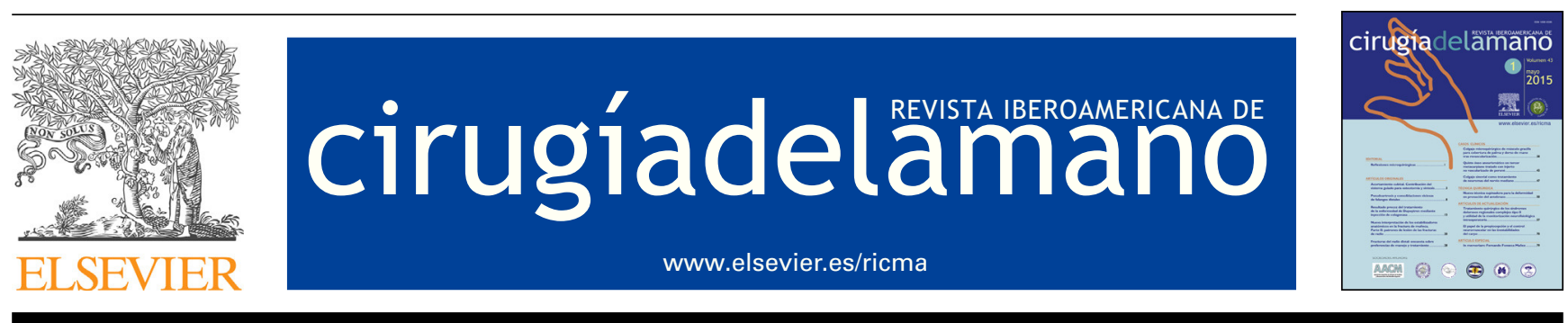

ARTÍCULO ORIGINAL

\title{
Rotura espontánea del aparato extensor secundaria a artrosis de la articulación radiocubital distal
}

\author{
M. Cruz Sánchez*, G. Pidemunt Moli y J. Cebamanos Celma
}

Unidad de Cirugía de la Mano, Parc de Salut Mar, Barcelona, España

Recibido el 13 de julio de 2015; aceptado el 29 de septiembre de 2015

Disponible en Internet el 3 de octubre de 2015

\section{PALABRAS CLAVE \\ Rotura atraumática; Tendón extensor; Artrosis radio-cubital distal; \\ Transferencia tendinosa}

\begin{abstract}
Resumen
Objetivo: Presentar los resultados funcionales de pacientes no reumáticos, intervenidos por roturas del aparato extensor con artrosis de la articulación radiocubital distal. Valorar la influencia de la varianza y la luxación dorsal del cúbito en estos pacientes.

Material y método: Estudio retrospectivo de los casos de rotura atraumática del aparato extensor a nivel de la articulación radio-cubital distal intervenidos en el periodo 2002-2012. Se recogen variables sociodemográficas, estudio radiológico centrado en análisis de factores desencadenantes: artropatía, morfología de la articulación, presencia de subluxación de la cabeza cubital y varianza cubital. Se descarta la enfermedad reumática mediante estudio analítico. Se detalla la técnica quirúrgica realizada según la presencia de factores desencadenantes y el número de tendones afectos. Se valora el resultado funcional por medio de la escala TAM, Quick-DASH y el dolor según la escala EVA.

Resultados: Se identifican 8 casos de rotura espontánea del aparato extensor por artrosis en la articulación radio-cubital distal. Media de edad 73 años (66-81). Seguimiento medio 5 años (212). En 7 casos se observa afectación tendinosa múltiple, principalmente los encargados de los dedos medio, anular y meñique. En el estudio radiológico destaca la presencia de una varianza cubital minus en 3 casos. Resultado funcional según escala TAM: bueno en 5 casos y regular en 3 casos. EVA media de 2. Quick-DASH 21.7.

Conclusiones: Los pacientes presentan a medio-largo plazo un resultado funcional aceptable utilizando la escala TAM y el cuestionario Quick-DASH, con un dolor residual leve según la escala EVA. Todos los pacientes mantienen o mejoran su TAM comparados con su exploración preoperatoria, obteniendo un resultado bueno en el $62.5 \%$ de los casos. No es necesaria la presencia de una varianza plus o subluxación de la cabeza para desencadenar la rotura puesto que se observan 3 casos con varianza minus y sin subluxación.

(C) 2015 SECMA. Publicado por Elsevier España, S.L.U. Este es un artículo Open Access bajo la licencia CC BY-NC-ND (http: //creativecommons.org/licenses/by-nc-nd/4.0/).
\end{abstract}

\footnotetext{
* Autor para correspondencia.

Correo electrónico: 99930@parcdesalutmar.cat (M. Cruz Sánchez).
} 


\section{KEYWORDS}

Atraumatic rupture;

Extensor tendon;

Distal radioulnar

osteoarthritis;

Tendon transfer
Spontaneous rupture of the extensor mechanism secondary to osteoarthritis of the distal radio-ulnar joint

\begin{abstract}
Objective: To present functional outcomes of surgery for ruptures of the extensor apparatus with osteoarthritis of the distal radio-ulnar joint in non rheumatic patients. To evaluate the influence of ulnar variance and dorsal dislocation of the ulna in these patients.

Material and method: A retrospective study of atraumatic rupture of the extensor apparatus at the distal radio-ulnar joint in the period 2002-2012. Radiological study focused on analyzing trigger factors were performed. The surgical technique was performed taking into account number of affected tendons and joint disease. Functional outcome and pain is assessed by the TAM, Quick-DASH, VAS scale.

Results: 8 cases of spontaneous rupture of extensor mechanism were identified due to osteoarthritis distal radio-ulnar joint. Mean age 73 years (66-81). Mean follow-up five years (2-12). In 7 cases multiple tendon involvement, mainly those responsible for the middle, ring and little fingers were seen. In the radiological study it was found the presence of an ulnar minus variance in 3 cases. Functional outcomes in the TAM scale were good in 5 cases and fair in 3 cases. EVA 2. Quick-DASH 21.7.

Conclusions: All patients reflect an acceptable functional outcome following TAM and QuickDASH questionnaire, with a slight pain according to the visual analog scale. All patients maintained or improved their TAM scale compared with preoperative examination, obtaining a good result in $62.5 \%$ of cases. The presence of a variance plus or subluxation of the head to trigger the break is not required due to 3 patients with variance minus and without subluxation suffered tendon rupture.

(c) 2015 SECMA. Published by Elsevier España, S.L.U. This is an open access article under the CC BY-NC-ND license (http://creativecommons.org/licenses/by-nc-nd/4.0/).
\end{abstract}

\section{Introducción}

La rotura de los tendones extensores de la mano a nivel de la articulación radio-cubital distal es un problema relativamente frecuente en pacientes con artritis reumatoide ${ }^{1}$. Sin embargo, es menos frecuente en alteraciones locales de la articulación, como las secundarias a artrosis $^{2}$, secuelas de fractura, deformidad congénita ${ }^{3}$ o enfermedad de Kienböck ${ }^{4,5}$.

Su aparición en pacientes no reumáticos se observa en los casos de muñecas artrósicas, tanto de causa traumática como no traumática ${ }^{6}$; ello desencadena la rotura secuencial del aparato extensor empezando por los tendones cubitales ${ }^{7}$. Pese a la trascendencia de la lesión y a que diversos autores han investigado su patogenia ${ }^{7,8}$, por el momento no hay publicaciones que evalúen el resultado funcional a medio-largo plazo. Se ha relacionado con diversos factores, entre ellos destaca la varianza cubital plus, aunque pocos autores han observado la varianza minus en el análisis radiológico de estos pacientes no reumáticos ${ }^{9}$.

El propósito del estudio es presentar el resultado funcional de una serie de casos de roturas espontáneas o por mínimo traumatismo del aparato extensor, y valorar la influencia de la varianza cubital, la luxación de la cabeza cubital y la presencia de osteofitos como factores clave en el abordaje de esta patología.

\section{Material y métodos}

\section{Criterios de inclusión y variables de estudio}

Se trata de un estudio retrospectivo en el cual se han incluido los casos de rotura espontánea del aparato extensor durante el periodo comprendido 2002 y 2012. Se identificaron 8 casos secundarios a artrosis en la articulación radio-cubital distal. En 2014 se realizó una valoración en consulta, donde se recogieron las características demográficas, cuestionario Quick-DASH ${ }^{10}$ y una exploración reglada donde se cuantificó la movilidad en grados de flexión y extensión de los dedos transformando el resultado funcional según la escala Total Active Motion (TAM) ${ }^{11}$. Se valoró el dolor cubital en la pronosupinación según la escala visual analógica (EVA). La estabilidad residual de la epífisis distal del cúbito se valoró según la aparición de un chasquido o resalte durante la pronosupinación con una carga de $2 \mathrm{~kg}$ en la mano. Se realizó una revisión de la historia clínica obteniendo los datos de la exploración clínica preoperatoria y del estudio radiológico.

En todos los casos de rotura por enfermedad degenerativa de la articulación radio-cubital distal se descartó la presencia de enfermedad reumática mediante analítica sanguínea. Para ello, el factor reumatoide debe ser negativo, así como la normalidad en marcadores inflamatorios, como la velocidad de sedimentación globular y la proteína $\mathrm{C}$ reactiva. 


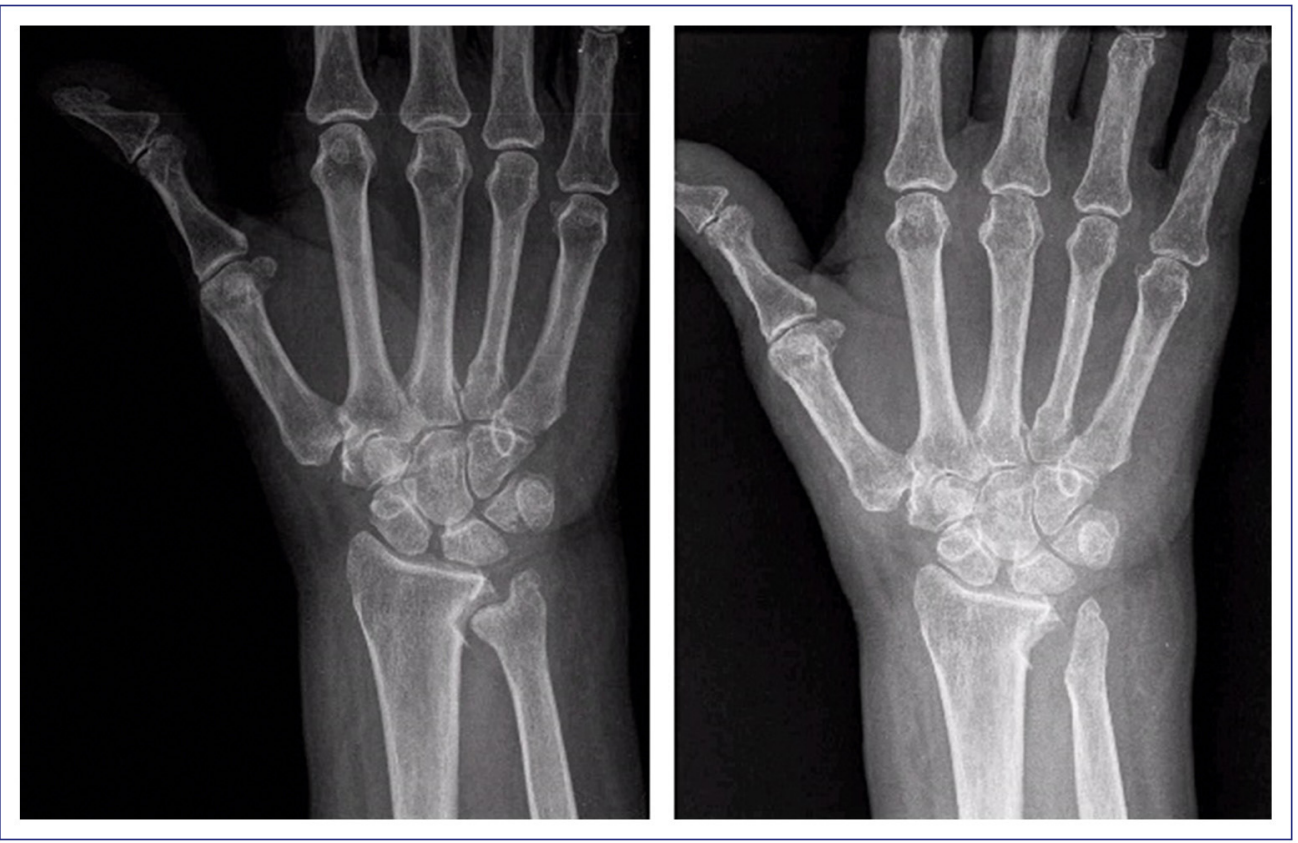

Figura 1 Radiología correspondiente al caso número 3: paciente afectado de artrosis radiocubital y varianza cubital minus. Se realizó técnica de Bowers.

El estudio radiológico preoperatorio constó de radiografía simple en proyecciones postero-anterior y lateral, donde se realizó la valoración de la morfología de la articulación radio-cubital, la presencia de subluxación dorsal de la cabeza cubital y el grado de artrosis según la escala de Kellgren-Lawrence ${ }^{12}$. La medición de la varianza ulnar ${ }^{13}$ se realizó midiendo la distancia entre la línea trazada desde el borde distal volar del radio y la cortical distal del cúbito mediante el sistema Picture Archiving and Communication System. La valoración de la presencia de subluxación dorsal de la cabeza cubital se realizó por medio de una proyección lateral pura (superposición del semilunar, polo proximal del escafoides y el piramidal, con la estiloides radial centrada sobre el radio distal). Además de la radiografía simple, también utilizó en todos los pacientes la tomografía computarizada como parte de la planificación preoperatoria, ya que permite una mejor valoración de los osteofitos dorsales tributarios de exéresis.

\section{Técnica quirúrgica}

La técnica quirúrgica realizada se basó en el tratamiento de los factores predisponentes a la rotura y la reconstrucción tendinosa. Para tratar los factores predisponentes, se realizó la técnica de Darrach ${ }^{14}$ en pacientes con escasa demanda funcional, incongruencia grave de la articulación radio-cubital distal o impactación cubital. En el resto de los casos se realizó una artroplastia de hemirreseccióninterposición ${ }^{15}$ (fig. 1). La reconstrucción tendinosa se basa en las transferencias tendinosas y en la utilización de injerto libre intercalado según el número de tendones a reparar. Los escenarios encontrados fueron desde la rotura del extensor digiti minimi (EDM), con escasa pérdida de extensión del quinto dedo, hasta roturas múltiples con afectación de

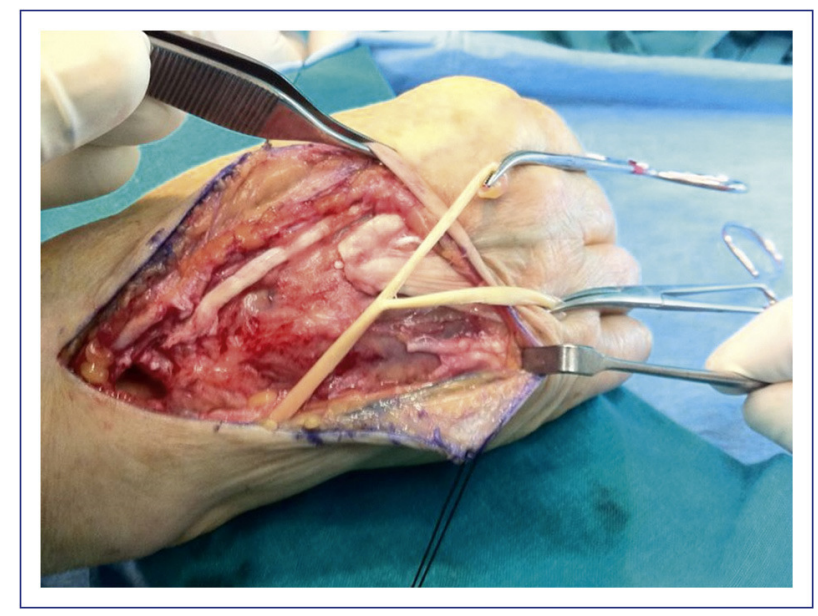

Figura 2 Rotura múltiple en la que se realiza una transferencia del flexor digital superficial del $4 .^{\circ}$ dedo.

3 dedos. Los casos más frecuentes fueron aquellos en los que se encontraron roturas dobles que afectaron al quinto y al cuarto dedo en donde el tratamiento utilizado fue la sutura lateral del cuarto al extensor digitorum communis (EDC) del tercero y una transferencia del extensor índicis proprius (EIP) al quinto ${ }^{16}$. En casos de roturas múltiples, se utilizó una transferencia del flexor digitorum superficialis (FDS) del cuarto dedo, pudiendo abastecer al cuarto y al quinto extensor una vez desdoblado (fig. 2). La utilización de injerto intercalar libre es una posibilidad que también presenta buenos resultados; la técnica más utilizada es el uso del palmaris longus con suturas tipo Pulvertaf. Un aspecto clave fue ajustar la tensión adecuada de la cuerda tendinosa; para ello, intraoperatoriamente se constató que dicha tensión permitía una flexión completa de la articulación 
metacarpofalángica con la muñeca en flexión. En ningún caso se realizó la sutura directa por la mala calidad de los cabos y el gap o defecto entre ellos. Respecto al procedimiento realizado en la articulación radiocubital distal, en 4 casos se optó por la técnica de Darrach y en 3 casos por la técnica descrita por Bowers. En un caso se realizó únicamente la resección de osteofitos a nivel de la epífisis distal del cúbito.

El protocolo rehabilitador postoperatorio consistió en una inmovilización mediante ortesis antebraquio-digital 2 semanas en posición de relajación del aparato extensor. Posteriormente, los pacientes se remitieron a fisioterapia para realizar movilización pasiva progresiva e inmovilización hasta la sexta semana mediante ortesis preconformada. A partir de la sexta semana postoperatoria, se permitió la movilización activa libre.

\section{Resultados}

Se identificaron 8 casos de rotura atraumática del aparato extensor debido a artrosis en la articulación radio-cubital distal. Se trató a 6 mujeres y 2 varones con una media de edad de 73 años (66-81) y sin antecedente de artritis reumatoide ni de fractura previa a nivel de radio o cúbito.

La rotura más frecuente fue la que afecta al EDM y EDC del $4 .^{\circ}$ y el $5 .^{\circ}$ dedos observada en 4 casos. En 3 casos, la rotura afectó al EDM, EDC del $3 .^{\text {er }}$, el $4 .^{\circ}$ y el $5 .^{\circ}$ (fig. 3). Solamente en un caso la rotura afectó a un único dedo: EDM y EDC del 5. (tabla 1$)$.

El estudio radiológico preoperatorio mostró en todos los casos que la artrosis en la articulación radio-cubital distal se asociaba a una alteración en la varianza cubital, observando cúbito plus en 5 casos o bien un cúbito minus en 3 casos. La presencia de osteofitos dorsales fue en 7 casos, mientras que la subluxación de la cabeza del cúbito se observó en 3 pacientes (tabla 2).

Existió una mejora de la escala TAM comparando los datos preoperatorios y postoperatorios en la última valoración realizada, en 5 casos bueno (> 75\% del balance articular comparado con contralateral) y en 3 casos regular $(50-75 \%$ de la función). El resultado medio del cuestionario QuickDASH fue de 21,78 (12-31,3). La valoración del dolor cubital fue de una EVA medio de 2 (0-3). La pronosupinación en los pacientes intervenidos con las 2 técnicas no se vio comprometida; únicamente cabe destacar que 2 casos intervenidos

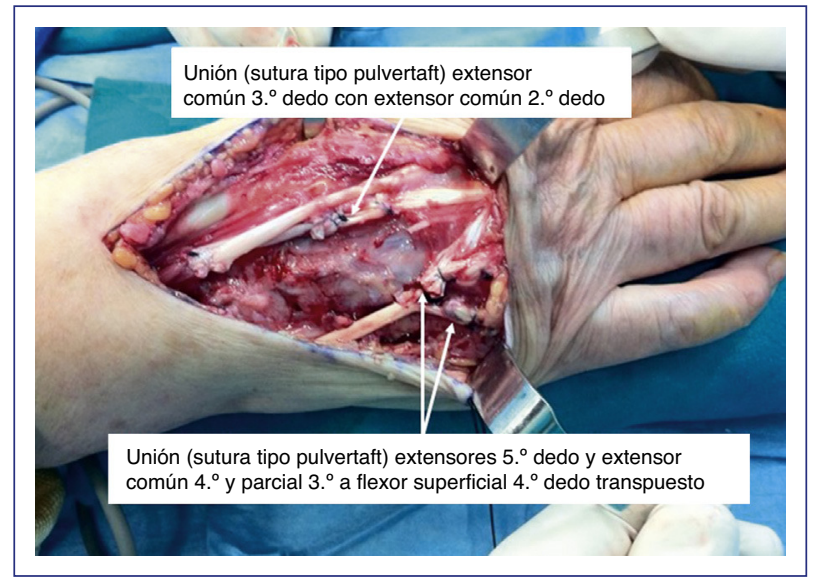

Figura 3 Rotura con afectación tendinosa del $3 .{ }^{\text {er }}$, el $4 .^{\circ}$ y el 5. ${ }^{\circ}$ dedos correspondiente al caso número 6 .

mediante resección cubital distal presentaban inestabilidad del muñón distal. No existió ningún caso de reintervención por nueva rotura al final del periodo de estudio, con 5 años de seguimiento medio (2-12).

\section{Discusión}

El primer resultado de este estudio es que los pacientes no reumáticos intervenidos por rotura del aparato extensor a nivel de la articulación radio-cubital distal secundaria a artrosis presentan a medio-largo plazo un resultado funcional aceptable. El resultado medio \pm desviación estándar del cuestionario Quick-DASH ha sido 21,78 , superior a los valores obtenidos en población general ${ }^{17}(10,1 \pm 14,68)$, aunque no excesivamente alejados. Sin embargo, todos los pacientes han mantenido o mejorado su escala TAM comparados con los datos preoperatorios, observando un resultado funcional bueno en el $62,5 \%$ de los casos. Hasta donde conocemos, no existen trabajos similares que estudien los resultados funcionales postoperatorios

El segundo resultado es constatar que no es necesaria la presencia de una varianza plus o subluxación de la cabeza para desencadenar la rotura ${ }^{8}$, como se podría esperar. Presentamos 3 casos en pacientes con cúbito minus y sin subluxación de la cabeza cubital que han presentado

Tabla 1 Resultado funcional según el tratamiento realizado y la lesión tendinosa previa

\begin{tabular}{|c|c|c|c|c|c|c|}
\hline & Edad (años) & Rotura & Tratamiento & $\begin{array}{l}\text { TAM } \\
\text { Preop. }\end{array}$ & $\begin{array}{l}\text { TAM } \\
\text { Postop. }\end{array}$ & Quick-DASH \\
\hline Caso 1 & 73 & 4,5 & Darrach + transferencia lat. & Regular & Regular & 19,3 \\
\hline Caso 2 & 67 & $3,4,5$ & $\begin{array}{l}\text { Bowers + } \\
\text { EPI + transferencia lat }\end{array}$ & Regular & Bueno & 27,5 \\
\hline Caso 3 & 72 & 4,5 & Bowers + transferencia lat. & Regular & Regular & 31,3 \\
\hline Caso 4 & 74 & 5 & $\begin{array}{l}\text { Exéresis osteofito + } \\
\text { transferencia lat }\end{array}$ & Bueno & Bueno & 12 \\
\hline Caso 5 & 71 & 4,5 & Darrach + transferencia lat & Regular & Bueno & 18,4 \\
\hline Caso 6 & 66 & $3,4,5$ & Bowers + FDS $4 .^{\circ}+$ transferencia lat. & Regular & Bueno & 22,1 \\
\hline Caso 7 & 81 & 4,5 & Darrach + transferencia lat. & Regular & Regular & 16,9 \\
\hline Caso 8 & 79 & $3,4,5$ & Darrach + EPI + transferencia lat & Regular & Bueno & 26,8 \\
\hline
\end{tabular}


Tabla 2 Estudio radiológico de preoperatorio

\begin{tabular}{lllll}
\hline & Kellgren-Lawrence & $\begin{array}{l}\text { Varianza } \\
(\mathrm{mm})\end{array}$ & Subluxación dorsal & Osteofito dorsal \\
\hline Caso 1 & 3 & +3 & $\mathrm{Sí}$ & $\mathrm{Si}$ \\
Caso 2 & 4 & -2 & No & $\mathrm{Si}$ \\
Caso 3 & 4 & -4 & No & $\mathrm{Si}$ \\
Caso 4 & 4 & +1 & $\mathrm{Si}$ & $\mathrm{No}$ \\
Caso 5 & 4 & +3 & $\mathrm{Sí}$ & $\mathrm{Si}$ \\
Caso 6 & 4 & -3 & No & $\mathrm{Si}$ \\
Caso 7 & 3 & +2 & No & $\mathrm{Si}$ \\
Caso 8 & 3 & +2 & No & $\mathrm{Si}$ \\
\hline
\end{tabular}

rotura tendinosa a nivel de la articulación radiocubital distal. Curiosamente, en todos ellos se ha observado la presencia de osteofitos dorsales a nivel intraoperatorio o en la tomografía computarizada.

Yamazaki ${ }^{9}$ estudia de forma pormenorizada los criterios radiológicos que se asocian a la rotura del aparato extensor y concluye que son un grado elevado de cambios artrósicos y la desviación dorso-radial de la cabeza cubital junto con una cavidad sigmoidea amplia y profunda (scallop sign). En cambio, no halla relación significativa con la morfología de la cabeza y la varianza positiva. La serie que se presenta concuerda con dichos criterios, ya que se ha observado casos de rotura con varianza cubital negativa, osteofitosis y subluxación de la cabeza cubital. La varianza cubital en su vertiente plus como minus podría ser un factor predisponente al desarrollo de artrosis primaria en la articulación radio-cubital distal, ya que se traduce una alteración biomecánica de la articulación ${ }^{18}$.

Para el tratamiento de la artropatía, diversos autores ${ }^{6-8}$ realizan la escisión de la cabeza cubital, sin definir la técnica de forma concreta ni su estabilización. En nuestra serie, en los casos de impactación cúbito-carpiana, y teniendo en cuenta la demanda funcional del paciente, se ha optado por la técnica de Darrach en 4 casos. En cambio, en los 3 casos con varianza ulnar negativa la técnica de elección ha sido la hemiartroplastia de resección e interposición debido a la mejor estabilización del cúbito distal.

Otra opción terapéutica es la implantación de una prótesis de cabeza cubital para el tratamiento de la artrosis y la inestabilidad de la articulación que se observa principalmente en el procedimiento de Darrach. Sin embargo, dado que el motivo de consulta es la rotura tendinosa y no el dolor, se puede reservar esta última opción para los casos que desarrollen un mal resultado en cuanto a dolor o estabilidad en la región cubital de la muñeca ${ }^{19}$. Para el uso de una prótesis se deben tener en cuenta la posibilidad de alargar el tiempo quirúrgico y sus complicaciones, por lo que se recomienda a cirujanos experimentados en su implantación.

Al igual que otros autores ${ }^{6,8}$, se ha podido constatar a nivel intraoperatorio la perforación que se produce en la cápsula, por la subluxación de la cabeza, y osteofitos que causan la atrición de la cuerda tendinosa. En estos casos, se debe realizar la estabilización del muñón y exéresis de los osteofitos.

Las opciones de tratamiento para la reparación tendinosa incluyen la sutura término-terminal, las transferencias tendinosas y el injerto intercalar ${ }^{20}$. La sutura término-terminal es a menudo imposible por el mal estado de los cabos y su retracción, lo que generaría una sutura de mala calidad con limitación para la flexión por la excesiva tensión ${ }^{6}$. Las transferencias tendinosas son preferibles al injerto por ser técnicamente más sencillas y con menor número de complicaciones ${ }^{20}$, dado que permiten resolver roturas múltiples mediante suturas laterales al EDC y el uso del EIP. En el caso de una rotura del EDM con escasa repercusión funcional se puede desestimar la reconstrucción, aunque es necesario vigilar que no progrese y se sucedan nuevas roturas, siendo en nuestra opinión preferible tratar la artropatía de forma profiláctica

No existen trabajos que describan el resultado funcional de la reparación de estas lesiones debido a la baja frecuencia y a la falta de uniformidad en el tratamiento. En nuestra serie, y teniendo en cuenta las limitaciones de un estudio retrospectivo, se ha conseguido otorgar función extensora intentando evitar la pérdida de flexión de los dedos; pese a que la puntuación es inferior a la de la mano no afectada, permite la realización de las actividades diarias con leve discapacidad, como refleja el cuestionario DASH. El leve dolor cubital postoperatorio y la ausencia de recidivas permiten mantener las directrices de tratamiento utilizadas para futuros casos.

\section{Conclusiones}

El resultado funcional de la reparación de la rotura atraumática del aparato extensor debido a artrosis de la articulación radio-cubital distal es bueno en términos de movilidad y dolor, con leve discapacidad para las actividades cotidianas. Para alcanzar estos resultados, es necesario tratar la artropatía y realizar una reconstrucción tendinosa por medio de transferencias o injerto libre.

La alteración en la varianza ulnar puede ser un desencadenante de la artrosis primaria que provoque la formación de osteofitos responsables de la atrición y rotura de la cuerda tendinosa.

\section{Conflicto de intereses}

Los autores declaran que no existe ningún conflicto de interés y que no han recibido ningún tipo de ayuda financiera para la realización del estudio. Su material únicamente ha sido expuesto como comunicación oral en un congreso nacional (SECOT) y que garantiza los derechos de privacidad y 
confidencialidad de los pacientes, así como su consentimiento para la publicación del manuscrito.

\section{Bibliografía}

1. Hernández Cortés P, et al. Rotura espontánea de los tendones extensores de la muñeca y la mano en pacientes con artritis reumatoide. Estudio retrospectivo. Rev Esp Reumatol. 2003;30(3):99-104.

2. Vaughan-Jackson OJ:. Rupture of extensor tendons by attrition at the inferior radio-ulnar joint: report of two cases. J Bone Joint Surg (Br). 1948;30B:528-30.

3. Ducloyer P, Leclercq C, Lisfranc R, Saffar P:. Spontaneous ruptures of the extensor tendons of the fingers in Madelung's deformity. J Hand Surg (Br). 1991;16:329-33.

4. Mazhar T, Rambani R. Vaughan-Jackson-like syndrome as an unusual presentation of Kienböck's disease: A case report. J Med Case Rep. 2011;5:325.

5. Pacha-Vicente D, Sevilla-Tirado J, López-Martínez R, LluchBergadà A, Mir- Bulló X, Llusá-Pérez M:. Extensor digiti minimi damage due to longstanding Kienböck's disease. J Hand Surg Eur. 2007;32:231-7.

6. Cho CH, Lee SW. Extensor tendon rupture caused by instability of the ulnar head with an osteoarthritic distal radioulnar joint: A case report. J Med Case Rep. 2013;7(1):281.

7. Ohshio I, Ogino T, Minami A, Kato H, Miyake A:. Extensor tendon rupture due to osteoarthritis of the distal radio-ulnar joint. J Hand Surg. 1991;16B:450-3.

8. Carr AJ, Burge PD:. Rupture of extensor tendons due to osteoarthritis of the distal radio-ulnar joint. J Hand Surg. 1992;17B:694-6.

9. Yamazaki H, Uchiyama S, Hata Y, Murakami N, Kato H. Extensor tendon rupture associated with osteoarthritis of the distal radioulnar joint. J Hand Surg Eur. 2008;33: 469-74.

10. Hudak PL, Amadio PC, Bombardier C. Development of an upper extremity outcome measure: The DASH (disabilities of the arm, shoulder and hand) The Upper Extremity Collaborative Group. (UECG).

11. Kleinert HE, Verdan C. Report of the Committee on Tendon Injuries (International Federation of Societies for Surgery of the Hand). J Hand Surg Am. 1983;8:794-8.

12. Kellgren JH, Lawrence JS. Radiological assessment of osteoarthrosis. Ann Rheum Dis. 1957;16(4):494-592.

13. Steyers CM, Blair WF. Measuring ulnar variance. A comparison of techniques. J Hand Surg. 1989;14A:607.

14. Huncherson DC. The Darrach operation for lower radioulnar derangement. Am J Surg. 1941;53:237-41.

15. Bowers WH. Distal radioulnar joint arthroplasty: The hemiresection-interposition technique. J Hand Surg Am. 1985;10:169-78.

16. Williamson SC, Feldon P. Extensor tendon ruptures in rheumatoid arthritis. Hand Clin. 1995;11:449-57.

17. Hunsaker FG, Cioffi DA, Amadio PC, Wright JG, Caughlin B. The American academy of orthopaedic surgeons outcomes instruments: normative values from the general population. J Bone Joint Surg Am. 2002;84(2):208-15.

18. Huang JI, Hanel DP. Anatomy and biomechanics of the distal radioulnar joint. Hand Clinics. 2012;28:157-63.

19. Van Schoonhoven J, Mühldorfer-Fodor M, Fernandez DL, Herbert TJ. Salvage of failed resection arthroplasties of the distal radioulnar joint using an ulnar head prosthesis: Long-term results. J Hand Surg Am. 2012;37:1372-80.

20. Tada H, Hirayama T, Takemitsu Y. Extensor tendon rupture after osteoarthrosis of the wrist associated with nonrheumatoid positive ulnar variance. Clin Orthop Relat Res. $1991 ;(262): 141-7$. 